\title{
Correlation between Amniotic Fluid Optical Density (AFOD) and functional maturity status of the newborn at caesarean delivery in GDM.
}

\author{
${ }^{1}$ Dr Samartha Ram H., ${ }^{2}$ Dr Shankar Ram H S., ${ }^{3}$ Dr Sandhya Ram S., \\ ${ }^{4}$ Dr. Rama Krishna Hanuman \\ ${ }^{1}$ MBBS.DGO. Consultant obstetrician \& Gynecologist Sandhya Ram Maternity Hospital, Katampazhipuram, \\ Palakkad, Kerala- India. PIN: 678633 \\ ${ }^{2}$ M.S. (Gen) Sandhya Ram Maternity Hospital Katampazhipuram, Palakkad, Kerala- India. PIN: 678633. \\ ${ }^{3}$ MBBS.DA Sandhya Ram Maternity Hospital Katampazhipuram, Palakkad, Kerala-India. PIN: 678633. \\ ${ }^{4}$ M.D. (Obgyn) Sankar Laparoscopy \& Infertility Center, Chirala, Andhra Pradesh-India. PIN: 523155
}

\begin{abstract}
:
Objective: To observe the correlation between Amniotic Fluid Optical Density (AFOD) and functional maturity status of the newborn at caesarean delivery in 16 women with Gestational Diabetes Mellitus (GDM). Methods: Amniotic Fluid (A.F) samples were collected while doing caesarean sections around 38+wks CRL gestation. Uncentrifuged fresh $A F$ samples were used for AFOD estimations with colorimeter at 650nm. HbAlc estimations were done for all women just before caesarean sections. These women received antenatal steroids as per the RCOG guidelines ${ }^{I}$. Babies were evaluated for functional maturity status in terms of APGAR scores, development of RDS after 5mnts, adherence of vernix on skin surface, and color of the skin. Birth weights were recorded by electronic weighing machine. Results:Fourteen of these 16 women, were observed to have mature $A F O D$ values $(0.98 \pm 0.27)$, at differentgestational ages (GA)ranging from $36 w+6 d$ to $39 w+6 d^{2}$, 3 . Twelve of these 14 women had good glycemic control with HbAlc values between 5.1 and 5.6.Two women had poor glycemic control with HbAlc values 7.0 and 7.1. All these babies were fully functionally mature, and their skin was mature pale brown in color with very little vernix, and none of them developed RDS. Birth weights ranged from $2.6 \mathrm{~kg}$ to $3.9 \mathrm{kgs}$. Two women had premature AFOD values $(<0.40)^{2,3}$ at $36 \mathrm{w}+6 \mathrm{~d}$ and $40 \mathrm{wks}$ gestation. Their glycemic control was good with HbAlc values 5.4 and 5.6 respectively. Both these babies had plenty of vernix caseosa on skin surface, and developed severe RDS with in $5 \mathrm{mnts}$ after delivery which required invasive and noninvasive ventilation support and surfactant therapy. Conclusion: The concept of individual term for each fetus ${ }^{4}$ is also applicable in women with GDM. AFOD value $0.98 \pm 0.27$ assures functionally mature babies who do not develop RDS irrespective of GA and glycemic control.Babies born with AFOD values $<0.40$ are functionallypremature irrespective of GA and arelikely to develop RDS which may require ventilator support.
\end{abstract}

Key Words: Individual term for each fetus, Amniotic fluid optical density (AFOD), Gestational diabetes mellitus (GDM)

\section{Introduction}

The incidence of GDM is progressively increasing in proportion to the increasing global burden of GDM. This problem is growing in alarming proportions necessitating universal screening in south Asian countries like India ${ }^{5}$. Results from Amniotic Fluid Optical Density (AFOD) research indicate, fetuses attain completion of functional maturity at AFOD value $0.98 \pm 0.28$, and go in for spontaneous labor at any time from $35 \mathrm{w}+\mathrm{d}$ to $42 \mathrm{w}+\mathrm{d}$, indicating individual term for each fetus $\mathrm{s}^{2,3,4}$. Babies can be functionally fully mature even at $35 \mathrm{w}+\mathrm{d}$ gestation if AFOD value is $0.98 \pm 0.28$. On the other hand babies can be functionally premature and develop RDS even at $40 \mathrm{wks}$ if AFOD value $<0.40^{2,3,6}$. Even in cases of GDM, it is not an uncommon observation to observe, few babies born around 37wks do not develop RDS, and few babies born even around 40wks develop severe RDS which may require ventilator support. Decision making regarding the time of delivery in pregnancies with GDM is a balancing act between unheralded fetal death on one side, and functional prematurity and development of RDS on the other side. Even though amniotic fluid L/S ratio estimation is the gold standard for lung maturity assessment, it is costly, cumbersome, time taking, and not available in every setting, and not being done regularly by everybody. On the other hand AFOD estimation is cheap, easily available in any setting, and the results can be obtained within few minutes and helps to make quick decisions. In this study we attempted to 
explore the possibility of utilizing mature AFOD values for decision making regarding the time of delivery in pregnancies with GDM.

\section{Methods}

Sixteen singleton pregnant women with GDM who underwent first trimester scan for CRL gestational age estimation, and who underwent caesarean sections at around 38 wks gestation as per the NICE guide lines ${ }^{7}$ and also for other different indications were included in this study. Informed and written consent was obtained from all subjects who participated in this study. This study confirms to standards of declarations of Helsinki. While doing cesarean section careful hysterotomy was performed avoiding injury to bulging membranes. With $2 \mathrm{ml}$ disposable syringe fitted with $2.5 \mathrm{~cm}$ long $23 \mathrm{G}$ needle, the membrane was pierced and the AF sample was drawn. Blood stained and meconium stained $\mathrm{AF}$ samples were excluded from study. Un-centrifuged fresh $\mathrm{AF}$ samples were used for AFOD estimation by colorimeter at $650 \mathrm{~nm}$ wave length. These women received antenatal steroidsas per the RCOG guidelines ${ }^{1}$.

\section{Method of measuring AFOD}

The colorimeter was set $650 \mathrm{~nm}$ wave-lengths. The test tube containing distilled water (control solution) was inserted in to the cuvette holder of the machine and ' 0 ' reading was adjusted. Then the test tube containing fresh uncentrifuged A.F sample was inserted, and with a press of a button, the AFOD value was directly read from the display screen of the machine.HbA1c estimations were done for all women just before caesarean sections. APGAR scores were recorded at $1 \mathrm{mts}$ and at $5 \mathrm{mts}$. Babies were observed for functional maturity status in terms of RDS after five minutes of birth, and adherence of vernix caseosa on skin surface, and color of the skin. Birth weights were recorded by electronic weighing machine. The details of gestational age at delivery, AFOD values, HbAlc values, APGAR scores, birth weights, color of the skin, and development of RDS in each subject are shown in Table.1

\section{Results}

Among these sixteen women, 14 women with case numbers 1 to 14 in table. 1 had mature AFOD values $(0.98 \pm 0.27)$. These mature AFOD valueswere observed at caesarean sections done at different gestational ages ranging from $36 \mathrm{w}+6 \mathrm{~d}$ to $39 \mathrm{w}+6 \mathrm{~d}$. Birth weights ranged from $2.6 \mathrm{~kg}$ to $3.9 \mathrm{~kg}$. Among these 14 women, 12 women (case numbers 1 to 12 ) had HbAlc values ranging from 5.1 to 5.6, and in two women (case numbers 13 and 14) the values were 7.1 and 7.0.All these babies were fully functionally mature with Apgar score 9 at $1 \mathrm{mnt}$ and at $5 \mathrm{mnts}$, and none of them developed RDS. Their skin was mature pale brown in color with very little or no vernix caceosa adherent on their skin surface.

Two women, case numbers 15 and 16 had premature AFOD values $(<0.40)$. In case number 15 , the G.A at delivery was $40 \mathrm{wks}$, the AFOD value was 0.33 , and the HbA1c value was 5.6 and the birth weight was $3.3 \mathrm{~kg}$. There was plenty of vernix caseosa on skin surface. We didnot administer antenatal steroids for this woman as the pregnancy prolonged beyond 39wks. This baby developed severe RDS within 5mts after delivery, but responded well for oxygen supplementation by noninvasive ventilation for 3hour.

In case number 16 , the G.A at delivery was $36 \mathrm{w}+6 \mathrm{~d}$, the AFOD value was 0.11 , and the HbA1c value was 5.4 and the birth weight was $2.7 \mathrm{~kg}$. She received two courses of antenatal steroids for recurrent preterm labor at $33 w+5 d$ and at $35 w+3 d$. The second course was given 10 days before caesarean delivery. This baby was functionally premature with plenty of vernix caseosa on skin surface. The skin was thin premature pink in color, and developed severe RDS with in 5mnts after delivery which required multiple doses of surfactant, and invasive and noninvasive ventilator support for 5days (Fig.1).

Table: 1. Details of GA at delivery, AFOD values, HbA1c values, APGAR scores, development of RDS, birth weights, skin color, and vernix on skin surface in each subject.

\begin{tabular}{|c|c|c|c|c|c|c|c|c|c|}
\hline \multirow{2}{*}{$\begin{array}{l}\text { Case } \\
\text { No }\end{array}$} & \multirow[b]{2}{*}{ G.A } & \multirow[b]{2}{*}{ AFOD } & \multirow{2}{*}{$\begin{array}{c}\text { HbA1c } \\
\text { before LSCS }\end{array}$} & \multicolumn{2}{|c|}{ APGAR at } & \multirow[b]{2}{*}{ RDS } & \multicolumn{2}{|l|}{ Birth wt } & \multirow{2}{*}{$\begin{array}{l}\text { Vernix on } \\
\text { skin surface }\end{array}$} \\
\hline & & & & $1 \mathrm{mnt}$ & $5 \mathrm{mnt}$ & & $\mathrm{Kg}$ & Skin colors & \\
\hline 1 & $37 w+5 d$ & 0.99 & 5.4 & 9 & 9 & Nil & 3.2 & mature pale brown & nil \\
\hline 2 & $38 w+5 d$ & 0.94 & 5.2 & 9 & 9 & Nil & 3.0 & mature pale brown & nil \\
\hline 3 & $38 w+1 d$ & 1.02 & 5.4 & 9 & 9 & Nil & 2.9 & mature pale brown & nil \\
\hline 4 & $37 w+4 d$ & 1.20 & 5.3 & 9 & 9 & Nil & 3.8 & mature pale brown & nil \\
\hline 5 & $38 w+6 d$ & 1.19 & 5.2 & 9 & 9 & Nil & 3.2 & mature pale brown & nil \\
\hline 6 & $38 w+0 d$ & 0.95 & 5.5 & 9 & 9 & Nil & 2.8 & mature pale brown & nil \\
\hline 7 & $36 w+6 d$ & 1.02 & 5.3 & 9 & 9 & Nil & 2.6 & mature pale brown & nil \\
\hline 8 & $37 w+2 d$ & 0.81 & 5.1 & 9 & 9 & Nil & 2.7 & mature pale brown & nil \\
\hline 9 & $38 w+2 d$ & 1.29 & 5.5 & 9 & 9 & Nil & 3.1 & mature pale brown & nil \\
\hline 10 & $37 w+6 d$ & 1.00 & 5.2 & 9 & 9 & Nil & 2.5 & mature pale brown & nil \\
\hline
\end{tabular}


Correlation between Amniotic Fluid Optical Density (AFOD) and functional maturity status of the

$\begin{array}{rrrrrrrrr}11 & 37 w+6 d & 0.95 & 5.6 & 9 & 9 & \text { Nil } & 2.8 & \text { mature pale brown } \\ 12 & 37 w+5 d & 1.90 & 5.4 & 9 & 9 & \text { Nil } & 2.85 & \text { mature pale brown } \\ 13 & 39 w+6 d & 1.33 & 7.1 & 9 & 9 & \text { Nil } & 3.9 & \text { mature pale brown } \\ 14 & 39 w+3 d & 1.20 & 7.0 & 9 & 9 & \text { Nil } & 3.9 & \text { mature pale brown } \\ 15 & 40 w+0 d & 0.33 & 5.6 & 9 & 6 & \text { present } & 3.33 & \text { nil } \\ 16 & 36 w+6 d & 0.11 & 5.4 & 9 & 6 & \text { present } & 2.70 & \text { premature pink pink }\end{array}$

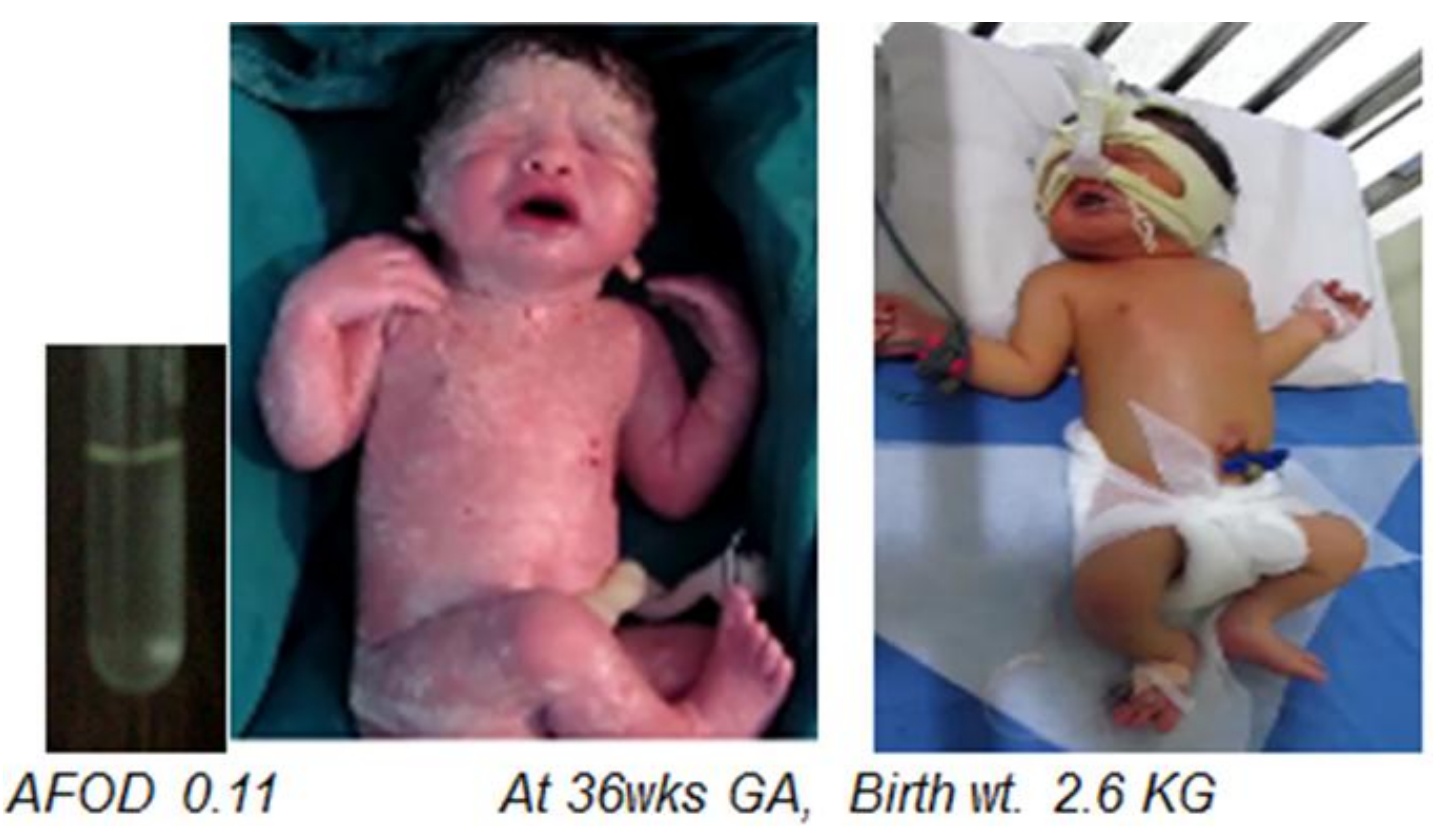

Fig.1: Newborn baby of a mother with GDM at $36 w+6 d$ GA (Case No.16). Functionally premature baby with plenty of vernix and with severe RDS. AFOD value was 0.11 (left). Baby was on bubble CPAP (Continuous

Positive Airway Pressure) on third post natal day in NICU (right).

\section{Discussion}

S. Ram.et.al reported, fetuses attain completion of functional maturity at AFOD value around 0.98 , and go in for spontaneous labor at any time from $35 \mathrm{w}+\mathrm{d}$ to $42 \mathrm{w}+\mathrm{d}$, indicating individual term for each fetus. Babies born with AFOD value $<0.40$ were functionally premature and develop varying degrees of RDS irrespective of GA and birth weight ${ }^{\mathbf{8}, 2,3}$. Babies can be functionally fully mature even at $35 \mathrm{w}+\mathrm{d}$ gestation if AFOD value is $0.98 \pm 0.28$. On the other hand babies can be functionally premature and develop RDS even at 40wks if AFOD value $<0.40^{2,3}$

In this study of sixteen women, 14 women with case numbers 1to 14 had mature AFOD values $(0.98 \pm 0.28)$, at different gestational ages ranging from $36 w+4 d$ to $39 w+6 d$. All these babies were fully functionally mature, and none of them developed RDS. It appears that, the concept of 'individual term for each fetus' is also applicable to women with $\mathrm{GDM}^{4,8}$.

Among these 14 women, 12 women (case numbers 1 to 12) had well controlled GDM with HbA1c values ranging from 5.1 to 5.6. In two women, case numbers 13 and 14, glycaemia was not under good control with $\mathrm{HbAlc}$ values 7.1 and 7.0. Their G.A at delivery was $39 w+6 \mathrm{~d}$ and $39 w+3 \mathrm{~d}$, and their AFOD values were 1.33 and 1.20 respectively. Both these babies were fully functionally mature and did not develop RDS. It appears that mature AFOD values assure functionally mature babies even in not well controlled GDM. This scientific information is having very much importance in the management of GDM.

In case numbers 15 and 16 , the G.A at delivery was $40 \mathrm{wks}$ and $36 \mathrm{w}+6 \mathrm{~d}$, and their glycemic control was very good with $\mathrm{HbA1c}$ values 5.6 and 5.4 respectively. The AFOD values were 0.33 and 0.11 , and their birth weights were 3.3 and $2.7 \mathrm{kgs}$ respectively. Both these babies had plenty of vernix caseosa on their skin surface and they developed severe RDS with in 5mnts after delivery. These results indicate that,irrespective of birth weights, GA at delivery, and even with good glycemic control, if babies born with AFOD values $<0.40$ are functionally premature and develop RDS.

In case number 15 we didn't administer antenatal steroids as the pregnancy was prolonged beyond $39 \mathrm{wks}$ as per the RCOG guidelines ${ }^{1}$. This baby had plenty of vernix caseosa on skin surface and developed severe RDS within $5 \mathrm{mts}$ after delivery, but responded well for oxygen supplementation by noninvasive ventilation for 3hour. It appears that, as the AFOD value in this case was very close to the mark of 0.40 , this baby responded well for simple measure of oxygen supplementation and other supportive measures. 
The woman with case number 16 received two courses of antenatal steroids for recurrent preterm labor at $33 w+5 d$ and at $35 w+3 d$. The second course was given 10 days before caesarean delivery. In spite of two courses antenatal steroids, this baby was functionally premature with plenty of vernix caseosa on skin surface, and developed severe RDS by 5mnts which required multiple doses of surfactant, and invasive and noninvasive ventilator support for 5days (Fig.1). Very low AFOD value (0.11) in this woman could be the reason for severe RDS which required all these treatment measures.

ACOG guidelines recommend the AFOD value $>0.15$ of a centrifuged amniotic fluid sample to confirm lung maturity, ${ }^{\mathbf{9} 10}$. Uncentrifuzed fresh AF sample with AFOD value around 0.40, when centrifuged at $2000 \mathrm{rpm}$ for $10 \mathrm{mnts}$ give a value of $0.15^{11}$. In other words an uncentrifuzed AFOD value $>0.40$ indicate completion of lung maturity. Skin is the last organ to mature which is associated with rapid shedding of vernix from fetal skin surface in to $\mathrm{AF}$ which results in rapid surge like rise in amniotic fluid optical density ${ }^{12,13}$, and4. The onset of spontaneous labor takes place at AFOD value around 0.98. In other words the AFOD value 0.98 can also be considered as a mark for completion of skin maturity. Between lung maturity and skin maturity, there is a period of 8 to 10 days, during which time preparations in cervix uterus and vaginatake place for the onset of spontaneous labor ${ }^{4}$.

NICE guidelines recommend, 'pregnant women with diabetes who have a normally grown fetus should be offered elective birth through induction of labor, or by elective caesarean section if indicated, after 38 completed weeks ${ }^{7}$. This recommendation is made to prevent unheralded fetal deaths in GDM.

Based on the above scientific information, it is advisable to perform simpler AFOD estimation at around $38 \mathrm{wks}$, instead of gold standard L/S ratio estimation which is costly, cumbersome, time taking and not available in every setting. This simple test helps to assess the functional maturity status of the fetus and also gives an idea about the number days further needed to attain the mature AFOD value of $0.98+/-0.27^{4}$. This information also helps to avoid indefinite waiting for the onset of spontaneous labor which may result in postmaturity, dysmaturity and unpredictable fetal deaths ${ }^{4}$. With mature AFOD value babies can be delivered without any further delay. In case of imminent labor with very low AFOD value like 0.11 , the women can be shifted to a higher medical center.

\section{Conclusion:}

The concept of individual term for each fetus is also applicable in women with GDM. AFOD value around 0.98 assures functionally mature babies who do not develop RDS irrespective of glycemic control. AFOD estimation around 38wks in GDM helps to assess the functional maturity status of the fetus and also gives an idea about the number days further needed to attain the mature AFOD value of $0.98+/-0.27$. This avoids unnecessary indefinite waiting for the onset of spontaneous labor which helps to prevent unpredictable fetal deaths. Babies born with very low AFOD value like 0.11 are likely to develop severe RDS which may require ventilator support irrespective of GA and glycemic control. AFOD estimation is cheap, easily available in any setting, and the results can be obtained within few minutes, and helps to make quick decisions when compared to $\mathrm{L} / \mathrm{S}$ ratio estimation. As this is a small study, these results should be further evaluated by multicentrec studies with larger sample size.

\section{References:}

[1]. Royal College of Obstetricians and Gynecologists, Antenatal Corticosteroids to Reduce Neonatal Morbidity and Mortality .Greentop Guideline No. 7. October 2010.

https://drive.google.com/file/d/0B9L0EY1ocMIkR1IPRV9pcFVRVVJMX0J0eUJNVEdINEo3b044/edit?usp=sharing

[2]. Ram S et al Amniotic fluid optical density at spontaneous onset of labor and its correlation with gestational age, birth weight, functional maturity and vernix caseosa of new born. Calicut Medical Journal 2009;7(4) Vol 7 Issue 42009 ... www.calicutmedicaljournal.org/2009/4/e2.pdf

[3]. Samartha Ram H, Sandhya Ram S, Amniotic fluid optical density (AFOD) surge coincides with the onset of spontaneous term labor. Paper presented at $55^{\text {th }}$ AICOG-2012. Varanasi, Book of abstracts: page 74. http://www.youtube.com/watch?v=-sjTQ0OX RA\&feature=g-upl

[4]. Samartha Ram H, Sandhya Ram S, Individualised Term for Each Fetus: From Surge in Amniotic Fluid Optical Density (AFOD). http://ispub.com/IJGO/18/1/14784

[5]. Purandare C. N. Universal Screening for Gestational Diabetes Mellitus (GDM): Mandatory. The Journal of Obstetrics and Gynecology of India. 2012; 62(2):141-143 http://www.researchgate.net/publication/236095281 Universal_Screening for Gestational Diabetes Mellitus (GDM) Mandatory

[6]. Husslein P, Salzer H, Simbrunner G. Pulmonary function of the newborn and surfactant level of amniotic fluid, following induced labour and spontaneous onset of labour (author's transl).Zentralblattfur Gynakologie [1980, 102(16):922-926] http://europepmc.org/abstract/MED/6893889

[7]. National Institute for Health and Clinical Excellence. NICE. Clinical Guideline 63: Diabetes in pregnancy. Management of diabetes and its complications from pre-conception to the postnatal period. London: NICE; 2008.

http://www.nice.org.uk/nicemedia/pdf/CG063Guidance.pdf

[8]. Shankar R Sandya R. Role of echogenic Amniotic Fluid particles and optical density in Prediction of RDS and Labor. Internet Journalof Medical Update 2010; 5(1):3-11

https://mail.google.com/mail/?ui=2\&ik=9edad23f89\&view=att\&th=1458cb4ca00b18c6\&attid=0.1\&disp=inline\&realattid=f_huc31 zde0\&safe $=1 \&$ zw

[9]. ACOG educational bulletin. ACOG Practice Bulletin No. 97: Fetal Lung Maturity. Obstet Gynecol. 2008;112(3):717-26. PMID: 18757686

https://drive.google.com/file/d/0B9L0EY1ocMIkOXRNNHJocmhWX013cWNBQ1RYbzFEeXZZMWM4/edit?usp=sharing 
[10]. Cetrulo CL, Sbarra AJ, Selvaraj RJ, Feingold M, Michlewitz H, Newton E,D’Alton ME, Herschel MJ, Kennedy JL, Shakr CJ. Positive Correlation between mature amniotic fluid optical density reading and the absence of neonatal hyaline membrane disease. $\mathrm{J}$ Reprod Med 1985; 30: 29-32.

http://www.ncbi.nlm.nih.gov/pubmed/4078829

[11]. Ram S, Sandhya, Ram S, et al. Amniotic fluid particles and optical density at term - a case report. The Internet Journal of Gynecology and Obstetrics. 2009;11(1): http://ispub.com/IJGO/11/1/9513

[12]. Joze H. Zabkar, Evaluation of fetal maturity by amnioscopy. J. Perinat. Med. 3 (1975) 145. https://drive.google.com/file/d/0B9L0EY1ocMIkUm14dXd2Nm9qRFV0QnJRYkY1OVRNV2O2M0pJ/edit?usp=sharing

[13]. Agorastos T, Vlassis G, Zournatzi B, Papaloukas A .Fetal lung maturity and skin maturity: 2 distinct concepts and the clinical significance of their differences.ZGeburtshilfePerinatol. 1983;187(3):146-50.

http://www.ncbi.nlm.nih.gov/pubmed/6688490 